\section{Parasitic Nematodes Recorded from Amphibians and Reptiles in Japan}

\author{
HIDEO HASEGAWA $^{1 *}$ AND \\ MITSUHIKO ASAKAWA ${ }^{2}$ \\ ${ }^{1}$ Department of Infectious Diseases \\ (Biology), Faculty of Medicine, Oita \\ University, Hasama, Oita 879-5593, \\ JAPAN \\ ${ }^{2}$ Department of Parasitology (Wildlife \\ Zoology), School of Veterinary Medicine, \\ Rakuno Gakuen University, Ebetsu, \\ Hokkaido, 069-8501, JAPAN
}

\begin{abstract}
A list of nematode species parasitic in amphibians and reptiles of Japan is presented. A total of 82 species are recorded, of which 51 are from amphibians, 42 from reptiles, and 11 from both groups. Three avian and six mammalian nematode species are known to utilize amphibians and reptiles as paratenic hosts.
\end{abstract}

Key words: Nematoda; Parasite; Amphibia; Reptilia; Japan; Species list

As a part of the species census in Japan under the auspices of the Union of Japanese Societies for Systematic Biology, we were assigned to count the number of parasitic nematode species hitherto known from Japan. The nematode species parasitic in freshwater fishes were already reviewed by Shimazu (1998), and those parasitic in birds and mammals were reviewed by Asakawa and Hasegawa (2003). Here we present a list of nematodes hitherto recorded from the Japanese amphibians and reptiles.

Early research on the nematode parasites of the Japanese herpetofauna was carried out by K. Morishita, S. Yamaguti, J. K. Wilkie, and A. Pearse during the first half of the 20th century. However, very little attention was

\footnotetext{
* Corresponding author. Tel: +81-97-586-5608; Fax: +81-97-586-5619;

E-mail address: hasegawa@med.oita-u.ac.jp
}

paid to taxonomic diversity and nomenclature in this group of organisms by subsequent researchers in Japan. Interest in nematodes parasitic in amphibians and reptiles was not strong among Japanese researchers. As a result, names of taxa invalidated outside Japan have been occasionally used in Japanese literature without critical reconsiderations (cf. Ishii, 1973; Kagei, 1973). Moreover, absence of type material has often caused difficulties in revising the taxonomy of problematic groups (cf. Schad, 1962; Sprent, 1978).

Checklists have been presented for helminth parasites of amphibians and reptiles of Japan several times (Kagei, 1973; Kamegai and Ichihara, 1973; Uchida, 1975, 1976). With respect to the nematodes parasitic in these vertebrates a comprehensive synopsis was also published by Baker (1987) for taxa of the world recognized then. However, these checklists are now outdated due to the results of subsequent research. Because of these taxonomic and nomenclatural problems, study of the nematode fauna in the Japanese amphibians and reptiles lags behind that in other vertebrate groups in Japan.

In listing nematode species here, we largely follow the molecular-based classification of De Ley and Blaxter (2002). However, this system is still incomplete in some details, and conflicts with traditional classification in the treatment of a number of taxa including several genera found in Japanese amphibians and reptiles. We have classified these genera provisionally.

Nematodes considered here are principally those having an adult stage in the amphibians and reptiles captured in Japan. Nematode species whose larval forms were recorded from these vertebrates are also listed, even though their adults are confined to other groups of hosts, such as mammals and birds. In recent years, various exotic amphibians and reptiles have been imported, often as pets, and some of them have established feral populations in the field (Ota, 1999; Lever, 2003). Nematodes reported from these introduced species are also considered in this study. In contrast, those found in exotic amphibians and reptiles 
in captivity or temporarily escaped from captivity are excluded (cf. Hasegawa and Katsuren, 1998).

Table 1 shows the nematode species hitherto recorded from the herpetofauna of Japan. The exact number of nematode species cannot be determined because identification of members of some genera is not reliable at the species level. Nevertheless, at least 82 nematode species are parasitic in the Japanese herpetofauna, of which 51 parasitize amphibians, 42 reptiles, and 11 both groups. Besides these, the larvae of three nematode species, which are parasitic in birds and six which are parasitic in mammals in the adult stage are known to utilize amphibians and reptiles as paratenic hosts. One species from amphibians and three from reptiles are recorded as pseudoparasites.

\section{LITERATURE CITED}

AsAKAWA, M. 2003. Faunal studies of parasites in Nopporo Forest Park-Ezo brown frog, Rana pirica-. Ann. Rep. Nopporo Forest (1): 31-36. (in Japanese)

Asakawa, M. AND H. Hasegawa. 2003. Parasitic nematodes from avian and mammalian species in Japan. Bull. Biogeogr. Soc. Japan 58: 79-93. (in Japanese)

BAKER, M. R. 1987. Synopsis of the Nematoda parasitic in amphibians and reptiles. Memorial Univ. Newfoundland Occ. Pap. Biol. (11): 1-325.

Bursey, C. R. AND S. R. GOLdBERG. 1999. Parapharyngodon japonicus sp. $\mathrm{n}$. (Nematoda: Pharyngodonidae) from the Japanese clawed salamander, Onychodactylus japonicus (Caudata: Hynobiidae), from Japan. Comp. Parasitol. 66: 180-186.

BurSEY, C. R. AND S. R. GOLdBERG. 2000. Angiostoma onychodactyla sp. n. (Nematoda: Angiostomatidae) and other intestinal helminths of the Japanese clawed salamander, Onychodactylus japonicus (Caudata: Hynobiidae), from Japan. Comp. Parasitol. 67: 60-65.

DE LEY, P. AND M. BLAXTER. 2002. Systematic position and phylogeny. p. 1-30. In: D. L. Lee (ed.), The Biology of Nematodes. Taylor and Francis, London.
GOlDBERG, S. R. AND C. R. BURSEY. 2002. Helminths of 10 species of anurans from Honshu Island, Japan. Comp. Parasitol. 69: 162-176.

GOLDBERG, S. R. AND C. R. BURSEY. 2003. Helminths of Ezo brown frog, Rana pirica (Ranidae), from Hokkaido Island, Japan. Pacific Sci. 57: 167-169.

GOlDBERG, S. R., C. R. BURSEY, AND H. CHEAM. 1996. Rana sakuraii (brown stream frog). Endoparasites. Herpetol. Rev. 27: 141.

GOLDBERG, S. R., C. R. BURSEY, AND I. RAMOS. 1994. Gastrointestinal helminths of the Japanese tree-frog, Hyla japonica (Anura: Hylidae), from Japan. J. Helminthol. Soc. Wash. 61: 225-227.

GoldBerG, S. R., C. R. BURSEY, AND Q. A. TRUONG. 1997. Helminths of Tago's brown frog, Rana tagoi tagoi (Ranidae) from Japan. Asiatic Herpetol. Res. 7: 44-47.

GoldberG, S. R., C. R. Bursey, AND S. R. TELFORD, JR. 2004. Helminths of six species of snakes from Honshu Island, Japan. Comp. Parasitol. 71: 49-60.

HASEGAWA, H. 1984. Helminth fauna of five Okinawan amphibian species. Biol. Mag. Okinawa (22): 11-22. (in Japanese)

HASEGAWA, H. 1985. Helminth parasites of reptiles from Okinawa, Japan. Biol. Mag. Okinawa (23): 1-11. (in Japanese)

HASEGAWA, H. 1986. Camallanus sp. collected from a frog, Rana limnocharis limnocharis in Oyama, Ginowan, Okinawa, Japan (Nematoda: Spirurida: Camallanidae). Akamata (3): 25-27. (in Japanese)

HASEGAWA, H. 1988. Parapharyngodon sp. (Nematoda: Pharyngodonidae) collected from the lizard, Ateuchosaurus pellopleurus (Sauria: Scincidae), on Okinawajima Island, Japan. Akamata (5): 11-14. (in Japanese)

HASEGAWA, H. 1989a. Nematodes of Okinawan amphibians and their host-parasite relationship. p. 205-217. In: M. Matsui, T. Hikida, and R. C. Goris (eds.), Current Herpetology in East Asia. Herpetological Society of Japan, Kyoto.

HASEGAWA, H. 1989b. Neoentomelas asatoi gen. et sp. n. (Nematoda: Rhabdiasidae) and Hedruris miyakoensis sp. n. (Nematoda: Hedruridae) from skinks of the Ryukyu Archipelago, Japan. Proc. Helminthol. Soc. Wash. 56: 145- 
150.

HASEGAWA, H. 1989c. Redescription of Cosmocercoides tridens Wilkie, 1930 (Nematoda: Cosmocercidae). Can. J. Zool. 67: 1189-1193.

HASEGAWA, H. 1990. Helminths collected from amphibians and reptiles on Amami-oshima Island, Japan. Mem. Natn. Sci. Mus. Tokyo (23): 83-92.

HASEGAWA, H. 1992a. Parasitic helminths collected from amphibians and reptiles on Kume-jima Island, Okinawa, Japan. Biol. Mag. Okinawa (30): 7-13. (in Japanese)

HaSEGaWA, H. 1992b. Parasites of the Iriomote cat, Felis iriomotensis (III). Island Stud. Okinawa (10): 1-24. (in Japanese)

HASEGAWA, H. 1993. Raillietnema rhacophori Yuen, 1965 (Nematoda: Cosmocercidae) collected from a frog, Polypedates leucomystax, on Okinawa-jima, Japan. Biol. Mag. Okinawa (31): 15-19.

Hasegawa, H., T. DoI, J. ARAKI, AND A. MiYATA. 2000. Kamegainema cingulum (Linstow, 1902) n. gen., n. comb. (Nematoda: Dracunculidae), a subcutaneous parasite of cryptobranchids (Amphibia: Caudata). J. Parasitol. 86: 583-587.

Hasegawa, H., T. DoI, AND A. Miyata. 1998. Spiroxys hanzaki n. sp. (Nematoda: Gnathostomatidae) collected from the giant salamander, Andrias japonicus (Caudata: Cryptobranchidae), in Japan. J. Parsitol. 84: 831-834.

Hasegawa, H., T. DOI, T. TOCHIMOTO, AND A. MiYATA. 2002. Parasitic helminths collected from the Japanese giant salamander, Andrias japonicus (Temminck, 1837) (Amphibia: Cryptobranchidae), in Japan. Comp. Parasitol. 69: $33-42$.

HASEgawa, H. AND N. Iwatsuki. 1993a. Prevalence of helminths in Rana narina in the foot of Mt. Yohana-dake, Okinawajima Island, in comparison with that in the upper region of the Benoki River, Akamata (8): 13-15. (in Japanese)

Hasegawa, H. AND N. Iwatsuki. 1993b. Prevalence of helminths in Bufo miyakonis on Miyakojima Island, Okinawa, Japan. Akamata (8): 16-18. (in Japanese)

HASEGAwa, H. AND S. KATSUREN. 1998. Helminth parasites found from cobras, Naja kaouthia, captured on Okinawa Island, Japan. Biol. Mag.
Okinawa (36): 19-27. (in Japanese)

Ishida, A., H. Iwao, M. AsaKawa, M. Hinoue, S. ABE, AND Y. KobAYASHI. 2004. Preliminary report of endoparasites obtained from 2 alien reptile species (Trachemys scripta and Chelydra serpentina) in Japan. Bull. Herpetol. Soc. Jpn. 2004: 35-36. (in Japanese)

ISHII, A. 1973. Internal parasites of the habu, Trimeresurus flavoviridis. Snake 5: 133-140. (in Japanese)

KAGEI, N. 1973. The endoparasites of snakes in Japan. Snake 5: 141-150. (in Japanese)

KAMEGAI, S. AND A. ICHIHARA. 1973. A check list of the helminths from Japan and adjacent areas. Part 2. Parasites of amphibia, reptiles, birds and mammals reported by S. Yamaguti. Res. Bull. Meguro Parasitol. Mus. (7): 33-64.

KoGA, M. AND Y. ISHII. 1981. Larval gnathostomes found in reptiles in Japan and experimental life cycle of Gnathostoma nipponicum. J. Parasitol. 67: 565-570.

KUZMIN, Y. I. 1999. Rhabdias agkistrodonis (Nematoda: Rhabdiasidae): morphology, distribution and specificity. Folia Parasitol. 46: 59-66.

LEVER, C. 2003. Naturalized Reptiles and Amphibians of the World. Oxford University Press, Oxford.

NAKACHI, A. AND H. HASEGAWA. 1992. Meteterakis ishikawanae Hasegawa, 1987 (Nematoda: Heterakoidea: Heterakidae), collected from the Japanese leaf turtle, Geoemyda spengleri japonica, on Okinawa-jima Island, Japan. Biol. Mag. Okinawa (30): 25-28.

OTA, H. 1999. Introduced amphibians and reptiles of the Ryukyu Archipelago, Japan. p. 439-452. In: G. Rodda, Y. Sawai, D. Chiszar, and $\mathrm{H}$. Tanaka (eds.), Problem Snake Management: The Habu and the Brown Treesnake. Cornell University Press, Ithaca, New York.

OTSURU, M. 1977. Angiostrongylus cantonensis. p. 343-374. In: M. Sasa (ed.), Animals of Medical Importance in the Nansei Islands in Japan. Shinjyuku-shobo, Tokyo.

SCHAD, G. A. 1962. Studies on the genus Kalicephalus (Nematoda: Diaphanocephalidae). II. A taxonomic revision of the genus Kalicephalus Molin, 1861. Can. J. Zool. 40: 1035-1165.

SHIMAZU, T. 1998. Nematodes of freshwater fishes 
in Japan: a review. J. Nagano Prefec. Coll. (53): 1-19. (in Japanese)

SPRENT, J. F. A. 1978. Ascaridoid nematodes of amphibians and reptiles: Polydelphis, Travassosascaris n. g. and Hexametra. J. Helminthol. 52: 355-384.

TELford, S. R., JR. 1997. The Ecology of a Symbiotic Community. Vols. 1 and 2. Population Biology of the Japanese Lizard, Tachydromus tachydromoides (Schlegel) (Lacercidae). Krieger Publ., Malabar, Florida.

UCHIDA, A. 1975. Check list of the helminth parasites of Japanese amphibians. Bull. Azabu
Vet. Coll. (30): 63-81. (in Japanese)

UCHIDA, A. 1976. Check list of the helminth parasites of Japanese amphibians (supplement). Bull. Azabu Vet. Coll. 1: 23-27. (in Japanese)

UCHIDA, A. AND H. ITAGAKI. 1979. Studies on the amphibian helminths in Japan. VI. Pseudoxyascaris japonicus n. g. and n. sp. (Oxyascarididae; Nematoda) and Pseudopolystoma dendriticum (Monogenea: Trematoda) from a salamander. Jpn. J. Parasitol. 28: 43-50.

Accepted: 27 April 2004

TABLE 1. A list of parasitic nematodes hitherto recorded from amphibians and reptiles of Japan (scientific name of parasite followed by host name and site in host in parenthesis, and reference in brackets).

\section{Phylum NEMATODA}

\section{Class ENOPLEA}

Subclass Dorylaimia

\section{Order Dioctophymatida}

Family Dioctophymatidae

Dioctophyme renale (Goez, 1782)* [larva] (Andrias japonicus; stomach wall) [Hasegawa et al., 2002]

\section{Order Trichinellida}

\section{Family Capillariidae}

Amphibiocapillaria bufonis (Morishita, 1926) (Bufo japonicus; small intestine) [Goldberg and Bursey, 2002]

Amphibiocapillaria tritonispunctati Diesing, 1851 (Hynobius nebulosus, H. stejnegeri, Onychodactylus japonicus, Andrias japonicus, Cynops pyrrhogaster, C. ensicauda; small intestine) [Baker, 1987; Hasegawa, 1989a, 1990; Hasegawa et al., 2002]

Aonchotheca buccalis Yamaguti, 1943 (Bufo japonicus; buccal cavity) [Uchida, 1975; Baker, 1987]

Aonchotheca sp. (Rana narina, R. amamiensis; buccal cavity) [Hasegawa, 1989a, 1990; Hasegawa and Iwatsuki, 1993a]

Paracapillaria kuntzi Moravec et Gibson, 1986 (Elaphe quadrivirgata, Rhabdophis tigrinus tigrinus; small intestine) [Goldberg et al., 2004]

Paracapillaria sp. (Rana narina; small intestine) [Hasegawa, 1989a; Hasegawa and Iwatsuki, 1993a]

Capillariidae gen. sp. (Trimeresurus flavoviridis, T. okinavensis; small intestine) [Hasegawa, 1985]

Capillariidae gen. sp. (Elaphe quadrivirgata; small intestine) [Kagei, 1973] 


\section{Class CHROMADOREA}

Subclass Chromadoria

Order Rhabditida

Suborder Spirurina

Superfamily Dracunculoidea

Family Dracunculidae

Kamegainema cingula (Linstow, 1902) (Andrias japonicus, skin) [Hasegawa et al., 2000]

Superfamily Gnathostomatoidea

Family Gnathostomatidae

Gnathostoma doloresi Tubangui, 1925* [larva] (Hynobius naevius, H. stejnegeri, Cynops ensicauda, Rana narina, $R$. subaspera, Amphiesma pryeri pryeri, Dinodon semicarinatum, Trimeresurus elegans, T. okinavensis, T. flavoviridis; viscera, muscle) [Kagei, 1973; Uchida, 1976; Hasegawa, 1985, 1990; Hasegawa and Iwatsuki, 1993a]

Gnathostoma nipponicum Yamaguti, 1941* [larva] (Rhabdophis tigrinus tigrinus; muscle) [Koga and Ishii, 1981]

Gnathostoma spinigerum Owen, 1836* [larva] (Rana catesbeiana, R. nigromaculata) [Uchida, 1975]

Spiroxys hanzaki Hasegawa et al., 1998 (Andrias japonicus; stomach) [Hasegawa et al., 1998, 2002]

Spiroxys japonica Morishita, 1926 (Rana japonica, $R$. nigromaculata, $R$. rugosa; duodenum, stomach) [Goldberg and Bursey, 2002]

\section{Superfamily Oxyuroidea}

Family Pharyngodonidae

Parapharyngodon japonicus Bursey et Goldberg, 1999 (Onychodactylus japonicus; rectum) [Bursey and Goldberg, 1999]

Parapharyngodon sp. (Ateuchosaurus pellopleurus, Eumeces marginatus marginatus; rectum) [Hasegawa, 1985, 1988]

Pharyngodon polypedatis Yamaguti, 1941 (Buergeria buergeri; rectum) [Goldberg and Bursey, 2002]

Skrjabinodon sp. or spp. (Gekko hokouensis, Gehyra mutilata, Hemidactylus frenatus, $H$. bowringii, Polypedates leucomystax; rectum) [Hasegawa, 1985, 1990]

Pharyngodonidae gen. sp. (Rhacophorus viridis viridis; rectum) [Hasegawa, 1989a]

Pharyngodonidae gen. sp. (Rhacophorus viridis amamiensis; rectum) [Hasegawa, 1989a (referred to as Skrjabinodon sp.)]

\section{Superfamily Camallanoidea}

\section{Family Camallanidae}

Camallanus sp. (Rana limnocharis; rectum) [Hasegawa, 1986]

Serpinema intermedius (Hsü et Hoeppli, 1931) (Chinemys reevesii; intestine) [Baker, 1987]

Serpinema microcephalus (Dujardin, 1845) (Trachemys scripta; stomach) [Ishida et al., 2004]

\section{Superfamily Physalopteroidea}

Family Physalopteridae

Abbreviata sp. (Dinodon rufozonatum walli, Trimeresurus elegans; stomach) [Hasegawa, 1985]

Pseudabbreviata yambarensis Hasegawa et Otsuru, 1984 (Japalura polygonata polygonata; duodenum) [Hasegawa, 1985] 
Physalopteridae gen. sp. (Andrias japonicus ${ }^{\dagger}$, intestine) [Hasegawa et al., 2002]

\section{Superfamily Spiruroidea}

Family Spirocercidae

Ascarops strongylina (Rudolphi, 1819)* [larva] (Japalura polygonata polygonata; stomach wall) [Hasegawa, 1992b]

\section{Superfamily Habronematoidea}

Family Hedruridae

Hedruris ijimai Morishita, 1926 (Rana japonica, R. nigromaculata, R. rugosa; duodenum) [Uchida, 1975; Baker, 1987]

Hedruris miyakoensis Hasegawa, 1989 (Scincella boettgeri, Bufo gargarizans miyakonis; stomach) [Hasegawa, 1989b; Hasegawa and Iwatsuki, 1993b]

\section{Family Cystidicolidae}

Spinitectus ranae Morishita, 1926 (Rana nigromaculata, $R$. rugosa; stomach) [Uchida, 1975; Baker, 1987]

\section{Superfamily Acuarioidea}

\section{Family Acuariidae}

Dispharynx nasuta (Rudolphi, 1819) ${ }^{\S}$ [larva] (Buergeria japonica, Microhyla ornata, Rana limnocharis, Gekko hokouensis, Hemidactylus frenatus, Japalura polygonata polygonata, etc.; alimentary canal wall, abdominal muscle, etc.) [Hasegawa, 1984, 1985]

Dispharynx sp. ${ }^{\S}$ [larva] (Buergeria japonica, Microhyla ornata, Rana limnocharis, Gekko hokouensis, Hemidactylus frenatus, Japalura polygonata polygonata, etc.; alimentary canal wall, abdominal muscle, etc.) [Hasegawa, 1984, 1985, 1992a]

Acuariidae gen. sp. ${ }^{\S}$ [larva] (Tachydromus smaragdinus; abdominal muscle) [Hasegawa, 1985]

\section{Superfamily Filarioidea}

\section{Family Onchocercidae}

Icosiella kobayashii Yamaguti, 1941 (Rana limnocharis; subcutaneous tissue, connective tissue) [Uchida, 1975; Baker, 1987]

Icosiella sasai Hayashi, 1960 (Rana subaspera; connective tissue) [Hasegawa, 1990]

\section{Superfamily Ascaridoidea}

\section{Family Ascarididae}

Hexametra quadricornis (Wedl, 1961) (Dinodon semicarinatum, Trimeresurus flavoviridis, T. okinavensis etc.; small intestine, stomach. Gekko hokouensis, Tachydromus tachydromoides; subcutaneous tissue, body cavity. Rana japonica, $R$. ornativentris, $R$. subaspera; body cavity) [Hasegawa, 1985, 1990; Telford, 1997; Goldberg et al., 2004]

\section{Family Raphidascarididae}

Paraheterotyphlum cf. ophiophagus Schmidt et Kuntz, 1973 (Laticauda semifasciata, esophagus) [Kagei, 1973]

\section{Superfamily Cosmocercoidea}

\section{Family Cosmocercidae}

Aplectana cf. macintoshi Stewart, 1914 (Rana ishikawae; rectum) [Hasegawa, 1990]

Cosmocerca japonica Yamaguti, 1938 (Rana japonica, $R$. nigromaculata, $R$. narina, $R$. ornativentris, $R$. rugosa, $R$. tagoi, Bufo japonicus, Hyla japonica, Microhyla ornata, Buergeria japonica, Polypedates leucomystax; Rhacophorus schlegelii, Cynops ensicauda; rectum) [Hasegawa, 1984, 1989a; Hasegawa and Iwatsuki, 1993a; Goldberg et al., 1994, 1996; Goldberg et al., 1997; Goldberg and Bursey, 2002] 
Cosmocercella iwatsukii Hasegawa, 1989 (Rhacophorus viridis viridis; rectum, small intestine) [Hasegawa, 1989a]

Cosmocercoides pulcher Wilkie, 1930 (Bufo japonicus, B. gargarizans miyakonis, Rana holsti, R. ishikawae, R. japonica, R. ornativentris; rectum. Elaphe quadrivirgata ${ }^{\dagger}$, Rhabdophis tigtinus $^{\dagger}$; small intestine) [Hasegawa, 1984, 1989a; Hasegawa and Iwatsuki, 1993b; Goldberg and Bursey, 2002, 2003; Goldberg et al., 2004]

Cosmocercoides tridens Wilkie, 1930 (Echinotriton andersoni, Cynops ensicauda; rectum) [Hasegawa, 1989a, 1989c, 1990]

Gyrinicola japonica Yamaguti, 1938 (Rana rugosa [tadpole]; intestine) [Uchida, 1975; Baker, 1987]

Raillietnema rhacophori Yuen, 1965 (Polypedates leucomystax; rectum) [Hasegawa, 1993]

Family Kathlaniidae

Falcaustra japonensis Yamaguti, 1935 (Mauremys japonica, Chinemys reevesii,? Mauremys mutica; rectum) [Baker, 1987; Hasegawa, unpublished observation]

Falcaustra sp. (Rana subaspera; small intestine) [Hasegawa, 1990]

Megalobatrachonema nipponicum Yamaguti, 1941 (Andrias japonicus; small intestine) [Hasegawa et al., 2002]

Pseudoxyascaris japonicus Uchida et Itagaki, 1979 (Onychodactylus japonicus; small intestine) [Uchida and Itagaki, 1979]

\section{Superfamily Heterakoidea}

Family Heterakidae

Meteterakis amamiensis Hasegawa, 1990 (Rana ishikawae, Ateuchosaurus pellopleurus, Eumeces marginatus oshimensis; rectum) [Hasegawa, 1990]

Meteterakis ishikawanae Hasegawa, 1987 (Rana ishikawae, Geoemyda japonica, Ateuchosaurus pellopleurus, Japalura polygonata polygonata; rectum) [Hasegawa, 1989a; 1992a; Nakachi and Hasegawa, 1992]

Meteterakis japonica (Wilkie, 1930) (Bufo japonicus, B. gargarizans miyakonis, Rana japonica, $R$. ornativentris, Eumeces latiscutatus, Tachydromus tachydromoides; rectum) [Hasegawa, 1984, 1989a; Hasegawa and Iwatsuki, 1993b; Telford, 1997; Goldberg and Bursey, 2002]

Meteterakis sp. (?Eumeces kishinouyei; ?rectum) [Hasegawa, 1992b]

\section{Family Ascaridiidae}

Strongyluris calotis Baylis et Daubney, 1923 (Japalura polygonata polygonata, J. polygonata ishigakiensis; lung, rectum) [Hasegawa, 1985 (referred to as Strongyluris japalurae (Yamaguti, 1935); 1992a]

\section{Superfamily Seuratoidea}

Family Seuratidae

Skrjabinelazia machidai Hasegawa, 1984 (Gekko hokouensis; intestine, stomach) [Hasegawa, 1985]

Family Quimperiidae

Wakubitinema toyamai Hasegawa, 1988 (Rana namiyei; small intestine) [Hasegawa, 1989a]

\section{Suborder Tylenchina}

\section{Superfamily Strongyloidoidea}

Family Strongyloididae

Strongyloides mirzai Singh, 1954 (Trimeresurus flavoviridis; small intestine) [Hasegawa, 1985] 
Strongyloides sp. or spp. (Chirixalus eiffingeri, Rana nigromaculata, Japalura polygonata ishigakiensis, Ateuchosaurus pellopleurus; rectum) [Hasegawa, 1985, 1989a, 1992a, unpublished observation]

\section{Family Rhabdiasidae}

Angiostoma onychodactyla Bursey et Goldberg, 2000 (Onychodactylus japonicus; rectum) [Bursey and Goldberg, 2000]

Entomelas cf. markovi Sczerbak et Sharpilo, 1969 (Tachydromus tachydromoides, Eumeces latiscutatus; lung) [Telford, 1997 (referred to as Rhabdias sp.); Hasegawa, unpublished observation]

Neoentomelas asatoi Hasegawa, 1989 (Ateuchosaurus pellopleurus; lung) [Hasegawa, 1989b, 1990, 1992a]

Rhabdias agkistrodonis Sharpilo, 1976 (Trimeresurus flavoviridis, T. okinavensis, ?T. elegans; lung) [Kuzmin, 1999]

Rhabdias horigutii Yamaguti, 1943 (Rhabdophis tigrinus tigrinus, Gloydius blomhoffii; lung) [Kagei, 1973; Baker, 1987; Goldberg et al., 2004]

Rhabdias incerta Wilkie, 1930 (Bufo japonicus, B. gargarizans miyakonis; lung) [Hasegawa, 1984; Hasegawa and Iwatsuki, 1993b; Goldberg and Bursey, 2002]

Rhabdias nipponica Yamaguti, 1935 (Hyla japonica, Rana japonica, R. limnocharis, $R$. narina, $R$. nigromaculata, $R$. ornativentris, ?R. pirica, $R$. tagoi, Buergeria buergeri, ?B. japonica; lung) [Hasegawa and Iwatsuki, 1993a; Goldberg et al., 1997; Goldberg and Bursey, 2002, 2003; Asakawa, 2002]

Rhabdias rhacophori Yamaguti, 1940 (Rhacophorus arborea, R. schlegelii; lung) [Uchida, 1975; Baker, 1987]

Rhabdias tokyoensis Wilkie, 1930 (Cynops pyrrhogaster, C. ensicauda; lung) [Hasegawa, 1984, 1990]

Rhabdias sp. (Japalura polygonata ishigakiensis; lung) [Hasegawa, 1985]

\section{Suborder Rhabditina}

\section{Superfamily Strongyloidea}

\section{Family Ancylostomatidae}

Kalicephalus brachycephalus Maplestone, 1931 (Amphiesma pryeri pryeri, Dinodon rufozonatum walli, Rhabdophis tigrinus tigrinus, Gloydius blomhoffii; rectum) [Hasegawa, 1985, 1992a; Goldberg et al., 2004]

Kalicephalus costatus indicus Ortlepp, 1923 (Amphiesma vibakari vibakari, Cyclophiops semicarinatus, Rhabdophis tigrinus tigrinus; small intestine) [Hasegawa, 1985; Goldberg et al., 2004]

Kalicephalus laticaudae Yamaguti, 1935 (Laticauda laticaudata, L. semifasciata; small intestine) [Kagei, 1973]

Kalicephalus posterovulvus Schad, 1962 (Amphiesma pryeri pryeri, Dinodon semicarinatum; esophagus) [Hasegawa, 1985, 1990]

Kalicephalus sinensis Hsü, 1934 (Elaphe climacophora, E. conspicillata, E. quadrivirgata; esophagus, small intestine) [Goldberg et al., 2004]

Kalicephalus viperae chungkingensis Hsü, 1934 (Trimeresurus okinavensis; esophagus) [Hasegawa, 1985, 1990]

Kalicephalus sp. (Dinodon rufozonatum walli; esophagus) [Hasegawa, 1985]

\section{Family Trichostrongylidae}

Batrachonema synaptospicula Yuen, 1965 (Rana narina, Rhacophorus viridis viridis; small intestine) [Hasegawa, 1989a; Hasegawa and Iwatsuki, 1993a] 
Oswaldocruzia insulae Morishita, 1926 (Bufo japonicus, B. gargarizans miyakonis, Hyla japonica, Rana rugosa; small intestine) [Hasegawa, 1989a; Hasegawa and Iwatsuki, 1993b; Goldberg et al., 1994; Goldberg and Bursey, 2002]

Oswaldocruzia hoepplii Hsü, 1935 (Rana limnocharis; small intestine) [Hasegawa, 1989a]

Oswaldocruzia cf. japalurae Jing et Lin, 1980 (Japalura polygonata ishigakiense; small intestine) [Hasegawa, 1985]

Oswaldocruzia socialis Morishita, 1926 (Rana japonica, $R$. nigromaculata, $R$. ornativentris, Rhacophorus arboreus, Rh. schlegelii, Bufo japonicus, Tachydromus tachydromoides, Elaphe quadrivirgata ${ }^{\dagger}$, Rhabdophis tigrinus $^{\dagger}$; small intestine, rectum) [Telford, 1997 (referred to as Oswaldocruzia filiformis); Goldberg et al., 1997; Goldberg and Bursey, 2002 2003; Goldberg et al., 2004]

Oswaldocruzia yezoensis Morishita, 1926 (Rana pirica, small intestine) [Asakawa, 2002]

Family Metastrongylidae

Angiostrongylus cantonensis (Chen, 1935* [larva] (Bufo gargarizans miyakonis, Rana limnocharis, R. catesbeiana, Polypedates leucomystax; liver) [Uchida, 1976; Otsuru, 1977]

${ }^{*}$ Adult stage parasitic in mammals.

$\S$ Adult stage parasitic in birds.

${ }^{\dagger}$ Pseudoparasitism. 